\title{
Recurrent Choledocholethiasis After Billroth II Gastrectomy: A Combined Endoscopic-Laparoscopic Technique
}

\author{
Li-Yun Zhang, MD, Nikolaus Zuegel, MD, Alain Schmit, MD, Martin Kox, MD \\ Centre Chirurgical, Centre Hospitalier Emile Mayrisch, Esch-sur-Alzette, Luxembourg (Drs. Zhang, Zuegel, Kox). \\ Department of Surgery, Ruijin Hospital, University School of Medicine, Shanghai, China (Dr. Zhang). \\ Department of Surgery, Campus Grosshadern LM-University Munich, Germany (Dr. Zuegel). \\ Department of Gastroenterology, Centre Hospitalier Emile Mayrisch, Esch-sur-Alzette, Luxembourg (Dr. Schmit).
}

\begin{abstract}
Background and Objective: After gastrectomy, the incidence of cholelithiasis formation increases. However, as a result of adhesions and reversed anatomy, either the surgery or the endoscopy is technically difficult. The aim of this article was to evaluate whether a combined endoscopic-laparoscopic "rendezvous" technique is effective in eliminating choledocholithiasis after Billroth II gastrectomy.

Methods: We present the case of an 89-year-old Caucasian man who had undergone appendectomy, laparoscopic cholecystectomy, and open distal gastrectomy with Billroth II gastrojejunostomy for early gastric cancer. He presented with recurrent choledocholithiasis and no fever or jaundice. Before admission, endoscopic retrograde cholangiopancreatography (ERCP) had been tried two times but was not feasible. Laboratory test findings revealed elevated cholestatic enzymes; magnetic resonance cholangiopancreatography revealed common bile duct stones. A laparoscopy was performed, and adhesions were lysed and jejunostomy was established by laparoscopy. A trocar was inserted and secured with a purse-string suture. The laparoscopically assisted ERCP was then performed.
\end{abstract}

Results: With the help of the laparoscopy, the papilla was reached successfully. Endoscopic sphincterotomy was performed. Common bile duct stones were extracted by irrigation/suctioning or with the help of a balloon catheter. Cholangiography was done to confirm complete stone clearance. A remarkable improvement was noticed, with normalization of liver function indexes. The patient was discharged home on postoperative day 6.

Conclusion: A combined endoscopic-laparoscopic rendezvous technique is an efficient, reliable, and minimally invasive method of treating choledocholithiasis after gastrectomy.

Key Words: Combined endoscopic-laparoscopic technique, Common bile duct stones, Laparoscopically assisted ERCP.

Citation Zhang L-Y, Zuegel N, Schmit A, Kox M. Recurrent choledocholethiasis after Billroth II. Gastrectomy: a combined endoscopic-laparoscopic technique. CRSLS e2014.00004. DOI: 10.4293/CRSLS.2014.00004.

Copyright (C) 2014 SLS This is an open-access article distributed under the terms of the Creative Commons Attribution-Noncommercial-ShareAlike 3.0 Unported license, which permits unrestricted noncommercial use, distribution, and reproduction in any medium, provided the original author and source are credited.

Drs. Zhang and Zuegel contributed equally to this study.

Address correspondence to: Nikolaus Zuegel, MD, Centre Chirurgical, Centre Hospitalier Emile Mayrisch, Esch-sur-Alzette, Luxembourg. Telephone: (+003) 52-621-296, Fax: (+003) 52-540-217, E-mail: nikolaus.zuegel@chem.lu

\section{INTRODUCTION}

Gastrectomy is known to increase the incidence of cholelithiasis. ${ }^{1,2}$ Some of these patients will become symptomatic, thus affecting their quality of life. However, either the surgical treatment or endoscopic application is tech- nically much more difficult because of the adhesions and reversed anatomy from their gastric resection. Our goal is to present a case of recurrent choledocholithiasis after Billroth II gastrectomy by laparotomy in which a combined endoscopic-laparoscopic "rendezvous" technique was successfully implemented. 


\section{Case Presentation}

An 89-year-old Caucasian male patient presented with a 1-year history of repeated right upper quadrant pain that had been aggravated in the prior 3 weeks; he had no fever or jaundice and had undergone appendectomy, laparoscopic cholecystectomy, and open distal gastrectomy with Billroth II gastrojejunostomy for early gastric cancer 17 years prior. The patient did not smoke or drink alcohol. Before admission, endoscopic retrograde cholangiopancreatography (ERCP) had been attempted twice but was not always feasible.

The patient appeared ill and had a blood pressure of $130 / 70 \mathrm{~mm} \mathrm{Hg}$, pulse rate of 86 beats/min, respirations of 20 breaths $/ \mathrm{min}$, and a temperature of $37.3^{\circ} \mathrm{C}$. A thorough physical examination revealed mild right upper quadrant tenderness on deep palpation without any other signs of peritonitis. The laboratory examination was unremarkable except for elevated cholestatic enzymes at the level of liver function. Specifically, alkaline phosphatase (ALP) counted $211 \mathrm{U} / \mathrm{L}$ (reference range, $10-129 \mathrm{U} / \mathrm{L}$ ) and $\gamma$-glutamyl transpeptidase (GGT) increased to $133 \mathrm{U} / \mathrm{L}$ (reference range, 5-66 U/L). Magnetic resonance cholangiopancreatography (MRCP) revealed common bile duct stones (CBDs) (Figure 1).

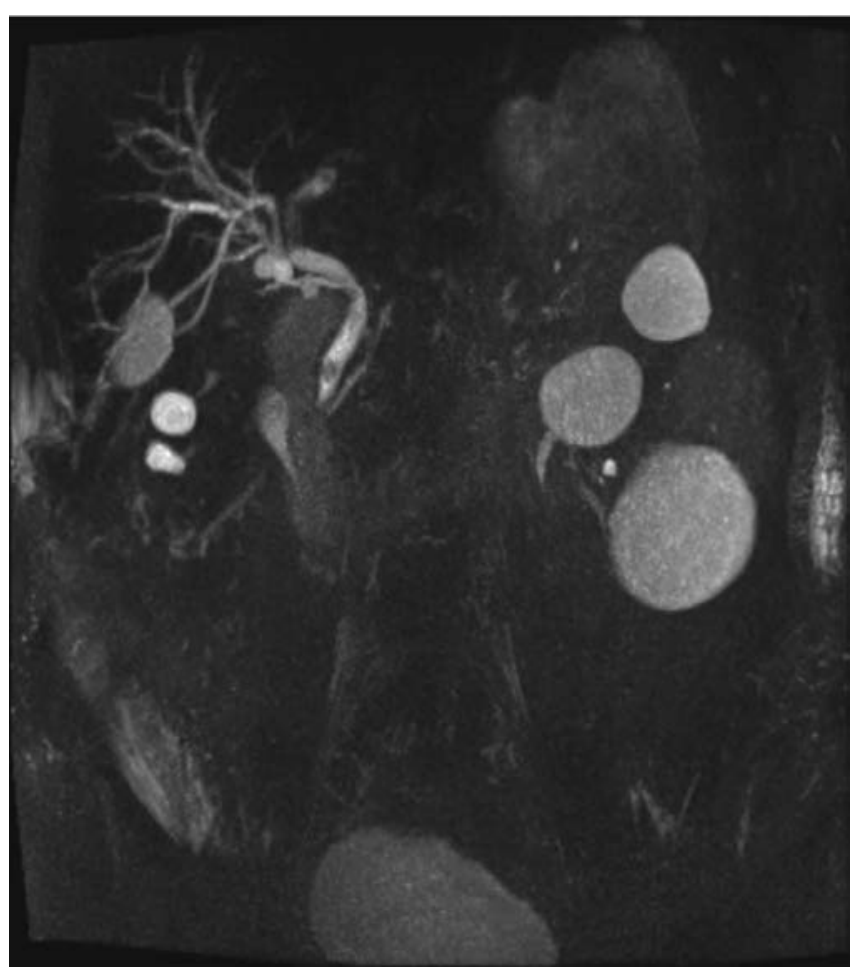

Figure 1. The MRCP showing CBDs.
A laparoscopic exploration was performed the next day. A pneumoperitoneum was established with carbon dioxide insufflations at a pressure of approximately $12 \mathrm{~mm} \mathrm{Hg}$. The extensive upper abdominal adhesions were observed. Two additional 5-mm ports were placed for laparoscopic adhesiolysis. A CBD dilated $>1 \mathrm{~cm}$ was found. A segment of jejunal export loop was sufficiently mobilized, and a $3-\mathrm{cm}$ left upper quadrant mini-laparotomy was performed, which was used for the creation of a jejunostomy. Through this incision, a purse-string suture was placed at the antimesenteric side of the jejunum. A jejunostomy was made with monopolar electrocautery, and a $15-\mathrm{mm}$ trocar was introduced into the lumen of the proximal bowel. The port was secured with the purse-string suture. An intestinal clamp was applied to the distal bowel for presentation of working space. The operative field was draped off, and a side-viewing duodenoscope was inserted through this port (Figure 2). The laparoscopically assisted ERCP then was performed. The papilla was reached with the help of the laparoscopy. Cannulation of the papilla and cholangiography revealed a dilated CBD and multiple stones in the CBD. Endoscopic sphincterotomy (EST) was performed. A balloon dilator was also introduced to dilate the CBD. Then CBD stones were extracted by irrigation/suctioning, or with the help of a balloon catheter. A completion cholangiogram was done to confirm complete stone clearance. Finally, the intestinal wall was sutured and the abdomen was closed, without endoscopic nasobiliary drainage (ENBD).

The postoperative course was uneventful, and the patient showed a significant and prompt recovery. Postoperative liver function indexes returned to normal. The patient was discharged home on postoperative day 6 .

\section{DISCUSSION}

The close relation to gastrectomy and postoperative cholelithiasis was first reported by Majoor et $\mathrm{al}^{3}$ in 1947 . After gastrectomy, the incidence of cholelithiasis formation has been reported in the literature with a high degree of variability, ranging from $18.8 \%$ to $31.0 \% .4,5$ Sapala et $\mathrm{al}^{6}$ found that, among all the factors, the type of gastrectomy, especially the type of gastric reconstruction, plays an important role in the occurrence of postoperative cholelithiasis. In detail, the non-physiological condition of Billroth II and Roux-enY anastomosis, without duodenal foodtransit, reduces the production of stomach acid and $\mathrm{fa}$ vours bacterial overgrowth. In consequence, components of bile also will be altered. Ultimately, it becomes a likely cause for stone formation. 


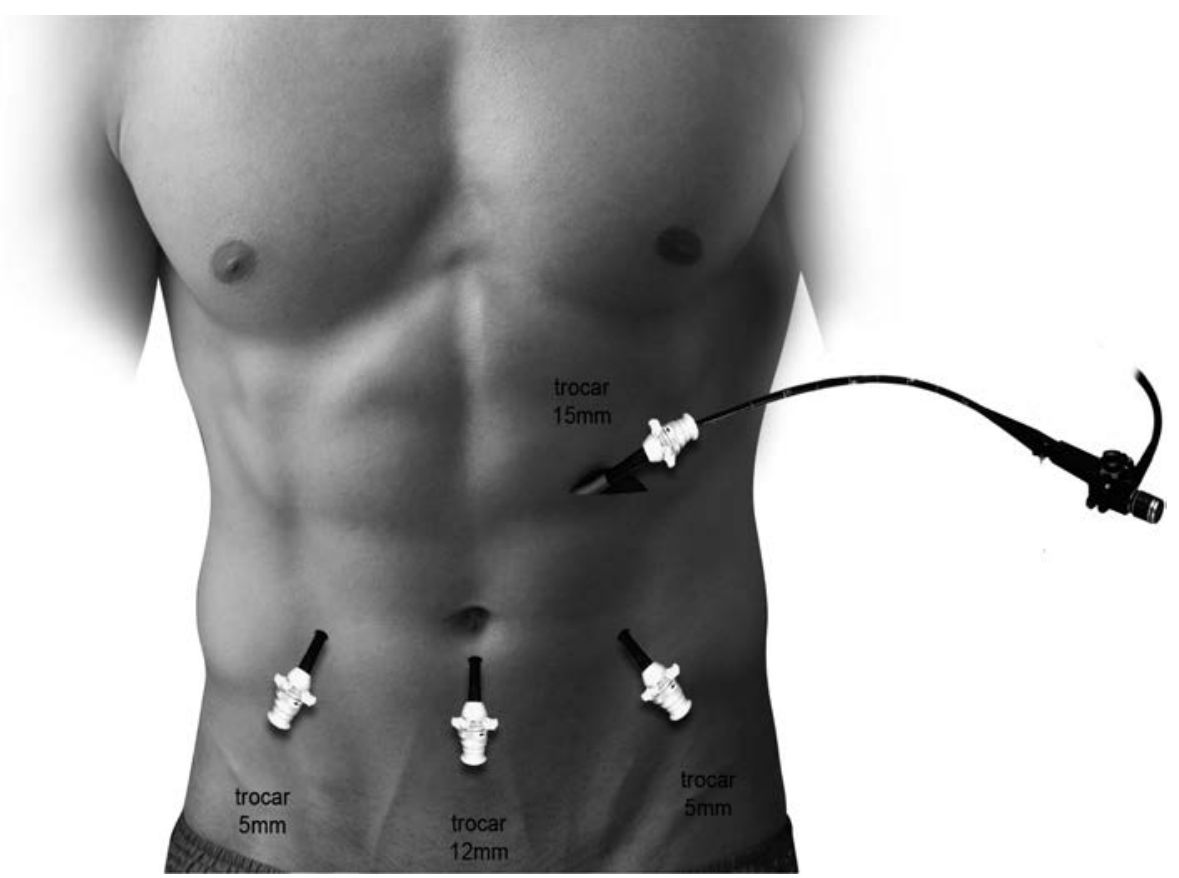

Figure 2. Port-sites.

It is reported that only $2 \%$ of patients with cholelithiasis after gastrectomy require surgery ${ }^{7}$; conversely, at present, ERCP is a commonly performed procedure by clinicians for the diagnosis and treatment of pancreaticobiliary disease, and the safety and utility of ERCP has been well documented. ${ }^{8}$

However, for patients with choledocholithiasis after gastrectomy, there are two main difficulties in terms of ERCP manipulation. First, the ability to advance the scope may be limited in patients with postoperative adhesions. Second, Billroth II and Roux-en-Y reconstruction exclude the biliary and pancreatic tree from traditional endoscopic evaluation and treatment with ERCP. The movement of the scope is thereby different from the usual.

In recent years, with laparoscopy being widely used in clinical diagnosis and treatment, it has become an accepted and favorite technique because of its expected low invasiveness, good cosmesis, and fast recovery. Meanwhile, a combined endoscopic-laparoscopic technique has gradually been developed. Lella et $\mathrm{al}^{9}$ reported that no cases of post-ERCP pancreatitis were observed in patients who had undergone the combined endoscopic-laparoscopic treatment, whereas 6 cases of acute post-ERCP pancreatitis occurred in a group of patients who had sequential endoscopic and laparoscopic treatment. Ghazal et $\mathrm{al}^{10}$ reported that a combined endoscopic-laparoscopic technique for the management of cholecystocho- ledocholithiasis is a safe and effective technique, with a low rate of post-ERCP pancreatitis. Nardi et al ${ }^{11}$ proposed that this technique may in the future be considered the treatment of choice for patients with cholecystocholedocholithiasis.

In our patient, the diagnosis of CBDs was clear, and ERCP had been already attempted two times before admission but was not feasible. Considering the patient's age and his previous abdominal surgeries, a combined endoscopic-laparoscopic rendezvous treatment was proposed above all. First, adhesions were lysed by laparoscopy to facilitate clarifying the relationship between bowels and mobilizing the jejunal export loop. Second, once a jejunostomy was established, a trocar was inserted and secured with a purse-string suture to avoid laceration of the intestinal mucosa. Next, the duodenoscope was advanced to the papilla with the help of the laparoscopy. This method allowed easier and faster cannulation, thus avoiding papillary edema and pancreatic trauma. Finally, the CBDs were removed by laparoscopically assisted ERCP.

\section{CONCLUSION}

This case prompts us that: (1) A combined endoscopiclaparoscopic rendezvous technique is an efficient, reli- 
able, and minimally invasive method of treating choledocholithiasis after gastrectomy; (2) in regard to reconstruction of the digestive tract after gastrectomy, if possible, the physiological reconstruction type, as the duodenal food passage, should be considered first.

\section{References:}

1. Rehnberg O, Haglund U. Gallstone disease following antrectomy and gastroduodenostomy with or without vagotomy. Ann Surg. 1985;201:315-318.

2. Hauters P, de Neve de Roden A, Pourbaix A. Cholelithiasis: a serious complication after total gastrectomy. Br J Surg. 1988; 75:899-900.

3. Majoor CLH, Suren TJJ. Gallbladder complications following resection of stomach for peptic ulcer. Br Med J. 1947;2:8-11.

4. Inoue K, Fuchigami A, Higashide S, et al. Gallbladder sludge and stone formation in relation to contractile function after gastrectomy. A prospective study. Ann Surg. 1992;215(1):19-26.

5. Pezzolla F, Lantone G, Guerra V, et al. Influence of the method of digestive tract reconstruction on gallstone development after total gastrectomy for gastric cancer. Am J Surg. 1993;166(1):6-10.
6. Sapala MA, Sapala JA, Soto AD, et al. Cholelithiasis following subtotal gastric resection with truncal vagotomy. Surg Gynecol Obstet. 1979;148(1):36-38.

7. Miyazaki I, Hashimoto T. Cholelithiasis following gastrectomy. Ryoikibetsu Shokogun Shirizu. 1996;(9):388-390.

8. Cohen S, Bacon BR, Berlin JA, et al. National Institutes of Health State-of-the-Science Conference Statement: ERCP for diagnosis and therapy, January 14-16, 2002. Gastrointest Endosc. 2002;56:803-809.

9. Lella F, Bagnolo F, Rebuffat C, et al. Use of the laparoscopicendoscopic approach, the so-called "rendezvous" technique, in cholecystocholedocho-lithiasis: a valid method in cases with patient-related risk factors for post-ERCP pancreatitis. Surg Endosc. 2006;20(3):419-423.

10. Ghazal AH, Sorour MA, El-Riwini M, et al. Single-step treatment of gall bladder and bile duct stones: a combined endoscopic-laparoscopic technique. Int J Surg. 2009;7(4): $338-346$.

11. Nardi M Jr., Perri SG, Pietrangeli F, et al. "Sequential" treatment: is it the best alternative in cholecysto-choledochal lithiasis? Chir Ital. 2002;54(6):785-798. 